\title{
Comparison between Linear Electromagnetic Accelerators
}

\author{
T. SIAENEN ${ }^{a, *}$ AND M.J. LÖFFLER ${ }^{b}$ \\ ${ }^{a}$ ISL, Saint-Louis, France \\ ${ }^{b}$ Gelsenkirchen University of Applied Sciences, Neidenburger Str. 10, D-45897 Gelsenkirchen, Germany \\ For symmetric Taylor tests a $2 \mathrm{~m}$ long electromagnetic accelerator will be used to accelerate $100 \mathrm{~g}$ rods up to \\ $300 \mathrm{~m} / \mathrm{s}$. Only a small variance of the muzzle parameters, velocity and exit time, is tolerable. In order to find the \\ most reliable, simple and efficient accelerator type, an axial coilgun, a flat-channel accelerator and an augmented \\ railgun are compared using a lumped parameter model. In particular, the accelerator mutual inductances and \\ their gradients characterize the propulsive forces. The essential advantages of the flat-channel geometry over the \\ axial coilgun geometry are shown. The geometric improvements of the flat-channel accelerator open the way for \\ the augmented railgun suitable and effective for the planned application. To minimize the variance of the muzzle \\ parameters, modular capacitor banks with semiconductor switches allow the dynamic control of the railgun \\ current, in principle.
}

PACS numbers: $85.70 . R p$

\section{Introduction}

With Taylor tests the mechanical properties of dynamically stressed materials are investigated [1]: A rod with planar ends hits a wall and deforms to a typical shape, giving an estimate of the dynamic material strain rate. Since the wall is not perfectly rigid and since friction losses distort the energy balance an error occurs in determining the stress-strain behaviour. Hence Erlich et al. [2] suggest a Symmetric Taylor Test (STT) in which the rod hits an identical one. Some electromagnetic accelerators (EMA) suit the STT requirements: limited constant acceleration to avoid rod deformation; reproducible impact velocity for the comparison of different experiments. An improvement of the STT is the Complete Symmetric Taylor Test (CSTT): two controlled EMAs are directed towards each other and accelerate the rods at the same time to guarantee an impact located between the muzzles. To find an EMA suitable for a (C)STT, different single-stage EMAs are compared: the axial coil gun (ACG), the flat-channel accelerator (FCA) [3] and the augmented railgun (ARG) $[4,5]$. At first specific properties of ACG and FCA (Fig. 1) are compared by using a lumped parameter model. In both systems the externally driven current $i_{1}$ in the inductor excites the current $i_{2}$ in the armature creating forces $F_{2}$ propelling the armature. For FCAs, the movement of the armature is in $z$-direction. The ACG shown in Fig. 1b works similar except that the armature moves in the $x$-direction. For direct comparison a square-shaped geometry is chosen rather than the usual circular geometry.

\footnotetext{
* corresponding author; e-mail: thorbjoern.siaenen@isl.eu
}

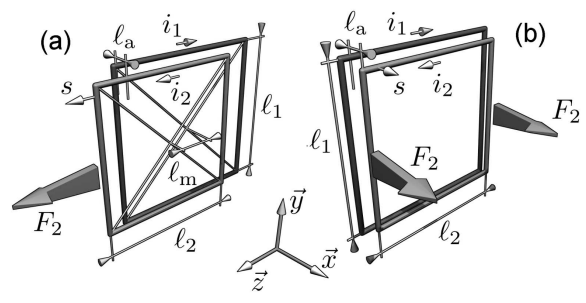

Fig. 1. (a) Sketch of the FCA. (b) Sketch of the ACG.

\section{Force and energy formulae}

The square-shaped inductor has the side length $\ell_{1}=$ $100 \mathrm{~mm}$ and $\ell_{2}=98 \mathrm{~mm}$ is the armature's side length. The conductors' circular cross section is $1 \mathrm{~mm}$ in diameter. For the ACG the centres of the inductor and of the armature coincide. The initial position is $\ell_{\mathrm{a}}=1.5 \mathrm{~mm}$. $s$ describes the movement of the armature. For the FCA the conductors keep a distance $\ell_{\mathrm{a}}=1 \mathrm{~mm}$ in the $x$-direction. The armature moves in the $z$-direction, $\ell_{\mathrm{m}}$ being the distance between both mid-points in this direction. The inductance of the inductor and of the armature is $L_{1}$ and $L_{2}$ respectively, the mutual inductance is given by $M_{12}$. Inductance formulae are taken from [6]. The magnetic coupling is expressed by $k=M_{12} /\left(L_{1} L_{2}\right)^{0.5}$ and varies between -1 and +1 . To get the same energetic efficiencies ACG and FCA have the same initial $k$ [7]. To obtain the same initial $k=0.68$, the armature moves by $2.18 \mathrm{~mm}$ in $z$-direction and $s$ is set to zero. Ohmic losses are neglected. The inductor is initially charged with $i_{1}(s=0)$. Thus the upper limits in terms of acceleration and kinetic energy transformation are investigated. Lenz's rule gives 


$$
i_{2}=-\frac{M_{12}}{L_{2}} i_{1} .
$$

Applying (1), the energy stored in the magnetic field yields

$$
E_{\mathrm{mag}}=i_{1}^{2} \frac{L_{1}}{2}\left(1-k^{2}\right) .
$$

With Lenz's rule, $i_{1,0} L_{1}=L_{1} i_{1}+M_{12} i_{2}$ gives the primary current

$$
i_{1}(s)=\frac{i_{1,0}}{1-k^{2}(s)},
$$

where $i_{1,0}$ denotes the current in the inductor when the armature is absent and $k=0$. Inserting (3) into (2) gives

$$
E_{\operatorname{mag}}(s)=i_{1,0}^{2} \frac{L_{1}}{2}\left[\frac{1}{1-k(s)^{2}}\right] \text {. }
$$

The force accelerating the armature is given by $F(s)=$ $-\nabla E_{\operatorname{mag}}(s)$ and yields:

$$
F(s)=-\frac{i_{1,0}^{2} L_{1} k(s)}{\left[1-k(s)^{2}\right]^{2}} \frac{\mathrm{d} k(s)}{\mathrm{d} s} .
$$

\section{Comparison}

The relative force $f_{\mathrm{rel}}(s)=F(s) /\left(i_{1,0}^{2} L_{1}\right)$ is shown in Figs. $2 \mathrm{a}$ and b. Figure 3 shows the functions of the coupling factor dependent on $s$.
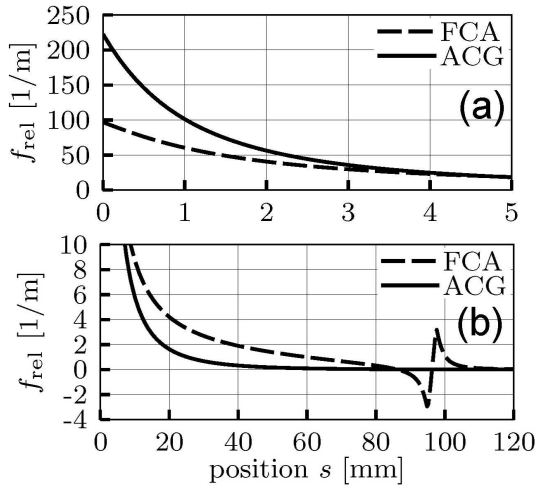

Fig. 2. (a) Relative force acting on the armature at different positions (detail). (b) Relative force acting on the armature at different positions.

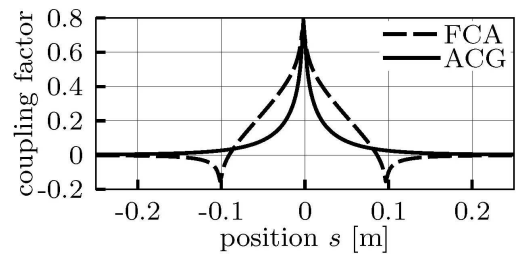

Fig. 3. Coupling factor of the FCA and the ACG depending on the position.

For the same initial primary current the force of the $\mathrm{ACG}$ is much higher than that of the FCA on the first $5 \mathrm{~mm}$. By contrast the FCA keeps a higher acceleration

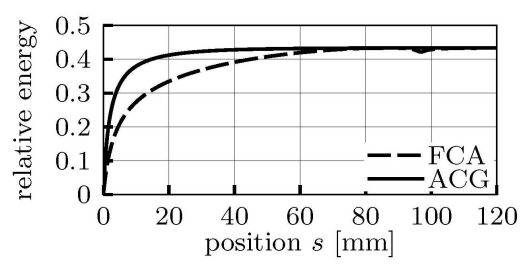

Fig. 4. Relative kinetic energy of the armature.

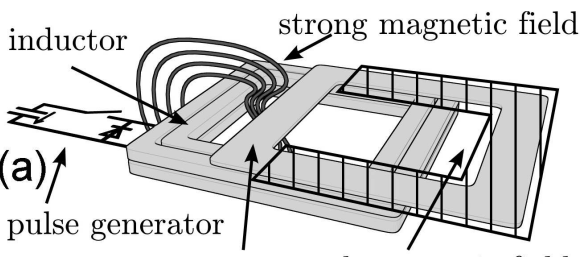

armature weak magnetic field

armature strong magnetic field

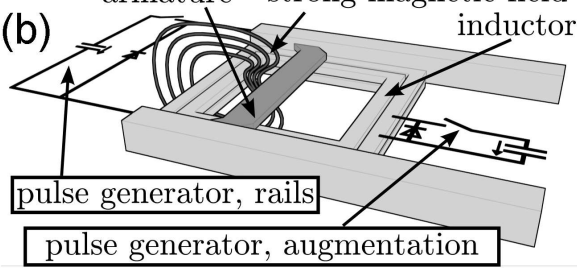

Fig. 5. (a) Sketch of an FCA. (b) Sketch of an ARG.

level when the armature moves by more than $10 \mathrm{~mm}$. The integral of this relative force over the covered distance gives the relative kinetic energy $e_{\text {rel }}$ at the position $s_{\text {end }}$

$$
e_{\text {rel }}\left(s_{\text {end }}\right)=\int_{0}^{s_{\text {end }}} f_{\text {rel }}(s) \mathrm{d} s .
$$

Figure 4 shows $e_{\text {rel }}$. Both cases reach the identical final value. Figure $2 \mathrm{a}$ shows that the initial force of the $\mathrm{ACG}$ is higher than that of the FCA. Hence the FCA outlined in Fig. 5 shows a better performance at a given acceleration limit. As the B-field is much stronger inside the coil than outside it, the back side of the armature produces most of the force. This is represented by the plain area in Fig. 5a. The only function of the striped area is to allow the circulation of the current. If the armature only consists of a single conductor with sliding contacts the arrangement equals an ARG with the inductor serving as an augmenting coil (Fig. 5b). This decreases the parasitic mass of the armature to $1 / 4$ compared to the FCA. There are additional advantages: Constant rail and armature currents yield a constant acceleration. Capacitors with semiconductor switches as used in the PEGASUS facility [8] allow controlling the acceleration, in principle. This control is easier than with ACGs or FCAs.

\section{Discussion and outlook}

In principle the FCA and the ACG show the same performance under identical initial conditions. However, for a given limit of acceleration FCAs show better performances. Improving the FCA immediately leads to the 
ARG with an essential decrease in the parasitic mass of the driving armature. Therefore, ARGs appear to be best suited for any of processes requiring a strong but limited and continuous acceleration such as the (C)STT. Furthermore, single-stage FCAs and ACGs have finite acceleration lengths, whereas the better controllable ARGs do not have this disadvantage, in principle. For the (C)STT an ARG will be used to accelerate $100 \mathrm{~g}$ rods up to $300 \mathrm{~m} / \mathrm{s}$. Slide contacts developed at ISL will guarantee the proper passage of the current through the rails into the armature.

\section{References}

[1] G.I. Taylor, A.C. Whiffin, Proc. R. Soc. A 194, 289 (1947).
[2] D.C. Erlich, D.A. Shockey, L. Seaman, AIP Conf. Proc. No. 78, 402 (1981).

[3] H. Weh, J. Jaquelin, F. Moisson, M. Löffler, Coilgun Concepts, 4th European Symposium on EM Launch Technology, Celle, DE (1993).

[4] J. Gallant, German-French Research Institute, Saint Louis, France, ISL-Report R 105/2005 (2005).

[5] M.J. Löffler, Ph.D. Thesis, Techn. Univ. of Braunschweig (1988).

[6] F.W. Grover, Inductance Calculations, Dover Publications, 1946.

[7] K. McKinney, P.P. Mongeau, IEEE Trans. Magn. 20, 239 (1984).

[8] P. Lehmann, H. Peter, J. Wey, IEEE Trans. Magn. 37, 235 (2001). 